\title{
SOBRE EL CONCEPTO DE LEY NATURAL*
}

JORGE BOSCH

Universidad Nacional de La Plata

\section{Planteo teórico general}

Sabido es que la expresión "ley natural" se usa en la literatura científico-filosófica con diversos sentidos, todos los cuales se hallan más o menos emparentados entre sí. En este artículo trato de caracterizar el concepto de ley natural en cuanto pauta del comportamiento de la naturaleza, por oposición al de ley natural en cuanto enunciado válido en el contexto de una teoría. La pregunta que me formulo es: "¿Qué aspecto tendría una ley que describiera el comportamiento real del universo?" Como se ve, es una pregunta que tiene una indudable componente ontológica. Pero tam. bién se ve que no es la versión más ontológica posible del problema de las leyes naturales. En efecto: el sentido ontológico más fuerte es el que concibe una ley natural como una entidad de existencia real en el universo, independientemen. te de toda formulación lingüística; una ley natural sería, según esta visión de las cosas, algo real que se manifestaría en el devenir de lo real: una necesidad inmanente, una fuerza concreta, un designio inscrito en la materia. Sin dejar de reconocer el alto valor especulativo y metafísico de este problema ontológico en sentido fuerte, no me ocuparé de él en este artículo. Resta un problema ontológico en sentido débil: aun admitiendo que una ley es un simple enunciado, es de.

* Una primera versión de este trabajo fue expuesta en las Jornadas Na. cionales de Lógica y Metodología de la Ciencia de la Univeraidad Nacional de Río Cuarto, el día 5 de diciembre de 1975; la presente versión fue expuesta en la Sociedad Argentina de Análisis Filosófico (SADAF) el 22 de mayo de 1976. En su redacción definitiva, he tenido en cuenta observaciones y sugerencias de Raúl Orayen. 
cir, una cierta entidad lingüística, ¿qué aspecto lógico-semántico-gramatical debe tener un enunciado para merecer que se lo califique como ley que describe el comportamiento del universo?

Este planteo nos conduce a establecer algunas condiciones necesarias para que un enunciado sea una ley natural. Tales condiciones, expresadas en un lenguaje informal y preliminar, son las siguientes:

(a) Veracidad: las leyes naturales deben ser enunciados verdaderos acerca del comportamiento de la naturaleza;

(b) Universalidad: las leyes naturales deben ser enunciados válidos en general, es decir, no restringidos a una determinada zona espacio-temporal del universo;

(c) No contingencia: las leyes naturales deben ser enunciados libres de todo matiz de contingencia. Por supuesto, mientras no aclaremos qué se entiende por "contingencia", esta condición carece de nitidez. Pero, precisamente, es mi deseo formular las condiciones que mi intuición exige a una ley natural, para tratar luego de establecer de manera más precisa cuál ha de ser la forma que deberá imponerse a las leyes naturales con el objeto de que se satisfagan las condiciones intuitivas previamente adoptadas. Es evidente que "libres de todo matiz de contingencia" puede significar lo mismo que "necesarios". Sin embargo, me parece un compromiso levemente menor poner énfasis en lo no contingente que ponerlo en lo necesario.

(d) Comunicabilidad: las leyes naturales deben ser enunciados teóricamente comunicables en el sentido siguiente: todo ser inteligente que conozca el significado de nuestros términos generales y que domine la gramática de nuestro lenguaje debe estar en condiciones de entender los enunciados que nosotros lla. mamos leyes naturales. 
Las tres primeras condiciones son de uso frecuente en la literatura relativa a leyes naturales. La condición $(d)$, en cambio, no es usual. La introduzco porque es la que apørta un matiz de conocimiento teórico en la caracterización de las leyes naturales, y ese matiz me parece tan importante como los otros. En virtud de las condiciones $(c)$ y $(d)$ se obtiene una conclusión bien conocida, a saber: que las leyes naturales no deben contener en forma esencial (esto es, no eliminable) términos que designen objetos particulares. En efecto: la aparición esencial del nombre de un objeto particular en una ley natural parece hacer depender el comportamiento del universo de la existencia de un objeto, lo cual puede ser considerado como una excesiva dosis de contingencia si se piensa - por ejemplo- que cada objeto aislado es en sí mismo contingente. Así, pues, la condición (c) nos llevaría a prohibir las menciones esenciales de objetos particulares; pero esta prohibición se ve reforzada notablemente por la condición $(d)$. En efecto: un objeto particular sólo puede ser inequívocamente identificado -en última instanciapor ostensión; y bien, la ostensión es, precisamente, una forma de comunicación que no puede ser efectuada a través de un texto teórico.

Por otra parte, en virtud de la condición $(b)$, no pueden figurar tampoco en forma esencial en el enunciado de una ley natural especificaciones de lugar o de tiempo. En cierto sentido esto es, a su vez, una consecuencia indirecta de la prohibición de usar términos que designen objetos particulares, pues las especificaciones espacio-temporales sólo pueden hacerse con respecto a un sistema particular de referencia, y tal sistema de referencia sólo puede individualizarse mencionando objetos particulares. Sin embargo, conviene mantener $(b)$ como condición independiente, por dos motivos: (1) si se aceptan el espacio y el tiempo absolutos el razonamiento que acabamos de hacer sobre los sistemas de referencia pierde validez; (2) se puede —al menos, teóricamente - establecer una especificación numérica espaciotemporal que no requiera la elección de un sistema de refe- 
rencia particular. Una tal especificación sería, por ejemplo: "velocidad mayor que $300,000 \mathrm{~km} / \mathrm{seg}$ en todo sistema de referencia (determinado salvo cambio de unidades)". Independientemente de que tales velocidades existan, esta especificación numérica es de carácter espacio-temporal, es perfectamente inteligible, y no requiere la elección de un sistema de referencia particular. Por cierto que hay en ella una referencia implícita a objetos particulares, a través de las unidades elegidas (kilómetro y segundo), pero lo que deseo mostrar con este ejemplo es que (aun en la hipótesis relativista) no toda especificación numérica de carácter espaciotemporal requiere la elección previa de un sistema de referencia privilegiado. Resta saber si hay ejemplos de especificaciones numéricas espacio-temporales que (asumiendo la hipótesis relativista) no requieren mención directa ni indirecta de objetos particulares; no conozco ningún ejemplo de esta indole, pero tampoco conozco ninguna argumentación conclusiva que pruebe su imposibilidad. (Por vía de mera conjetura, me inclino a pensar que tales ejemplos existen, aunque quizá en un contexto en el que se introduzca el mé. todo axiomático.)

\section{Condiciones básicas derivadas}

Llegamos así, partiendo de las condiciones $(a) \cdot(d)$ establecidas en $\S 1$, a las dos siguientes condiciones básicas derivadas, que han sido ampliamente discutidas en la literatura:

(e) No mención esencial de objetos particulares;

(f) No uso esencial de especificaciones numéricas de carácter espacio-temporal.

Todas estas condiciones - las primitivas $(a),(b),(c)$, $(d)$, y las derivadas $(e),(f)-$ son interesantes, pero no bastan. Surgen frente a ellas dos problemas fundamentales: el de los predicados puramente cualitativos, de Hempel-Oppenheim, y el de los accidentes cósmicos, de Popper-KnealeSimpson. Me ocuparé del primero de estos dos problemas 
en $\S 5$, y abordaré de inmediato el segundo, que me parece el más importante. Trataré de exponerlo haciendo uso de un clásico ejemplo de Popper, cuyas palabras transcribo:

Consideremos un animal extinguido, digamos la moa, un ave gigantesca cuyos huesos abundan en algunas ciénagas de Nueva Zelanda (yo mismo he excavado buscándolos). Decidimos utilizar el nombre de "moa" como nombre universal (en lugar de como nombre propio: cf. el apartado 14) de cierta estructura biológica; pero hemos de admitir que es completamente probable $\longrightarrow$ incluso completamente creíbleque no hayan existido en el universo moas nin. gunas, ni vayan a existir, excepto las que vivieron en otro tiempo en Nueva Zelanda; y asumamos que esta tesis creíble es exacta.

Supongamos ahora que la estructura biológica del organismo de la moa es de tal índole que un animal de esta especie pueda vivir fácilmente, en condiciones muy favorables, hasta sesenta años o más; y sụpongamos, además, que las condiciones con que se encontró la moa en Nueva Zelanda distaban mucho de ser ideales (debido, tal vez, a la presencia de cierto virus), de modo que ninguna moa llegó jamás a tener cincuenta años. En este caso, el enunciado estrictamente universal "todas las moas mueren antes de tener cincuenta años" será verdadero: pues, según los supuestos asumidos, no ha habido, hay, ni habrá moa en todo el universo con más de cin. cuenta años de edad. Pero este enunciado universal no será una ley de la naturaleza, pues -de acuerdo con las asunciones hechas - sería posible que una moa viviese durante más tiempo, y el hecho de que ninguna haya vivido más se debe únicamente a unas condiciones accidentales 0 
contingentes (tales como la copresencia de cierto virus). (Popper, [2], pág. 399.)

Podría haber —usando palabras de Kneale y de Simpson- un accidente cósmico en virtud del cual las moas están confinadas de hecho a una reducida zona del espacio-tiempo y tienen de hecho una vida de duración inferior a los cincuenta años; pero el ejemplo de Popper es altamente ilustrativo, como ha hecho notar Simpson, en el sentido siguien. te: lo que da la impresión de un verdadero accidente es la existencia de una ley biológica según la cual, en condiciones favorables, las moas podrian vivir más de cincuenta años (Cf. Simpson, [3]). Haciendo entonces un drástico resumen de lo señalado por Popper, Kneale (a quien tomo según cita de Simpson) y Simpson, se puede decir que un accidente cósmico es un hecho expresable mediante un enunciado estrictamente universal, cuyo carácter contingente es puesto en evidencia por una ley natural según la cual aquel hecho no se produciría en determinadas condiciones, objetivamente diferentes de las que realmente se dieron.

Las condiciones $(a)-(f)$ que hemos estipulado en $\$ \S 1$ y 2 no alcanzan a cerrar el camino a pretendidas leyes naturales que expresen accidentes cósmicos; y sin embargo nuestra intuición nos dice que la afirmación "todas las moas viven menos de cincuenta años" no es una ley natural. (Aclaremos, de paso, que el ejemplo tal cual lo presenta Popper adolece de pequeños defectos, pues hay en él una especificación temporal que requiere indirectamente la mención de objetos particulares: 50 años. Pero este detalle es fácilmente superable introduciendo un predicado tal como "joven", caracterizado por el estado de los tejidos, o de las arterias, etc.)

No creo que sea posible la caracterización individual de un enunciado que describa un accidente cósmico, porque la noción misma de accidente cósmico - tal como acabo de exponerla - requiere la introducción previa del concepto de ley natural. La situación parece ser circular: para caracterizar las leyes naturales debemos caracterizar previamente los 
enunciados que describen accidentes cósmicos, con el objeto de estipular que tales enunciados no pueden ser leyes naturales; pero para caracterizar los enunciados en cuestión debemos saber previamente qué es una ley natural.

Sin embargo, la circularidad es sólo aparente, y esta situación pseudo-circular nos indica el camino que debemos seguir para resolver el problema. Esbozaremos a continuación un plan basado en el concepto de "enunciado legaloide".

\section{Primera aproximación}

De lo expuesto surge que un plan razonable para resolver el problema sería el siguiente: (1) caracterizar, con auxilio de las condiciones $(a)-(f)$ establecidas en $\$ \S I$ y 2 , un concepto previo al de ley, que sería el de enunciado legaloide; (2) caracterizar, entre los enunciados legaloides, los que expresan accidente cósmico, ejemplificados paradigmáticamente por el ejemplo de las moas popperianas; (3) definir como ley natural a todo enunciado legaloide que no sea de accidente cósmico (y que no tenga otros inconvenientes menores). Lo que ofrezco a continuación es una primera aproximación a ese esquema, que aparenta traducir en términos formales las ideas intuitivas antes expuestas, y que permite poner de relieve las dificultades profundas, de carácter lógico-semántico, que se alzan en el camino del plan trazado.

3.1. Llamaremos enunciado legaloide a todo enunciado verdadero acerca de la realidad,' que satisfaga las condiciones $(e)$ y $(f)$ de $\$ 2$, y que, si es de la forma

$$
(x): P(x) \supset Q(x),
$$

no resulte fácticamente verdadero el enunciado $(x) \sim P(x)$. ( $O$ sea que, en el caso de que el enunciado legaloide tenga la forma de un condicional universal, su antecedente no sea universalmente falso. Esta condición elimina los candidatos a enunciados legaloides trivialmente $\longrightarrow$ vacíamente- verdaderos.) Prescindiremos por el momento de la objeción de 
Hempel-Oppenheim acerca de los predicados puramente cualitativos, que será comentada en $\$ 5$.

3.2. Un enunciado de accidente cósmico es un enunciado legaloide de la forma

$$
(x): \mathrm{M}(x) \supset \mathrm{J}(x)
$$

tal que, de hecho, la propiedad $M$ no es vacía (es decir que existe algún $x$ tal que $\mathbf{M}(x)$ ), y existen un enunciado legaloide $\mathrm{L}$ y una condición $\mathrm{C}$ no vacía, tales que de $\mathrm{L}$ se deduce

$$
(x): \mathrm{M}(x) \wedge \mathrm{C}(x) \cdot \supset \cdot \sim \mathrm{J}(x) \text {. }
$$

En el ejemplo de Popper, "M(x)" significa " $x$ es una moa", "J( $x)$ " es " $x$ muere joven (menos de cincuenta años)", $y$ " $\mathrm{C}(x)$ " es " $x$ vive en un ambiente carente de virus $\alpha$ "; se supone que tales ambientes existen en la realidad pero no se dieron precisamente en el caso de las maas; $L$ es un enunciado legaloide que hace las veces de la ley biológica que reclama Popper. Hay que hacer notar esta diferencia: Simpson (en su comentario sobre Popper) insiste en que L sea una ley biológica, mientras que en la versión que aquí propongo $L$ es solamente un enunciado legaloide; no se puede exigir que $\mathrm{L}$ sea una ley natural porque, precisamente, los enun. ciados de accidente cósmico se introducen para fundamentar la noción de ley natural. Es este matiz el que elimina la circularidad mencionada más arriba. La traducción del enunciado (B) al ejemplo popperiano sería: "si una moa vive en un ambiente carente de virus $\alpha$, entonces no muere joven (vive más de 50 años)". Aquí aparece una objeción menor, que consistiría en estimar que (B) es demasiado fuerte; en efecto, puede parecer excesivo que baste la ausencia de virus $\alpha$ para asegurar que la moa en cuestión vive más de $\mathbf{5 0}$ años. Se podría pensar que esto se corrige fácilmente debilitando de manera adecuada el enunciado (B) ; por ejemplo, poniendo en su lugar " $\sim(x): \mathrm{M}(x) \wedge \mathrm{C}(x) \cdot \supset \cdot \mathrm{J}(x)$ ". Sin embargo, este enunciado no es verdadero, por cuanto impli- 
ca la existencia de moas en condiciones $C$; otra posibilidad sería agregar condiciones al antecedente de $(B)$, pero esto da lugar a otras complicaciones técnicas. Como esto no es esencial, continuaremos trabajando con (B).

Obsérvese que entre (A) y (B) no hay contradicción lógica, pues de (A) se supone solamente que es fácticamente verdadero, y de (B) que es deducible de L. Es obvio que (B) es fácticamente verdadero porque el antecedente $\mathrm{M}(x) \wedge \mathrm{C}(x)$ es fácticamente falso para todo $x$, ya que si existiera un $x$ tal que $\mathrm{M}(x) \wedge \mathrm{C}(x)$ se cumpliría $\mathrm{J}(x)$ en virtud de $(A)$, y entonces (B) sería falso; luego, (B) no podría haber sido deducido de enunciados verdaderos. Pero, precisamente, no interesa la verdad fáctica de (B), que es trivial: lo que interesa es que (B) se pueda deducir de enunciados legaloides, los cuales son verdaderos en forma no trivial por definición. Esta deducibilidad de (B) a partir de L es lo que reemplaza a la exigencia de satisfacer un condicional subjuntivo, introducida por algunos autores.

3.3. Un enunciado legaloide redundarte es un enunciado legaloide de la formà

$$
(x): P(x) \wedge Q(x) \supset \mathbf{R}(x)
$$

tal que es fácticamente verdadero el condicional universal $(x): \mathbf{P}(x) \supset Q(x)$.

Esta versión de redundancia también puede parecer demasiado fuerte, o demasiado débil, según el punto de vista del que la juzgue; pero no vale la pena detenerse a analizarla, aun sospechando que sea defectuosa, pues se trata de una noción netamente secundaria y, en definitiva, prescindible.

3.4. Una ley natural es un enunciado legaloide que no es enunciado de accidente cósmico ni enunciado legaloide redundante.

3.5. Una ley natural pura es una ley natural que no es una ley lógica. 


\section{Objeción sobre la ontología y la verdad}

La definición de enunciado legaloide exige (ver 3.1) que un tal enunciado sea verdadero en cuanto hace referencia a la realidad. Entendemos por realidad (y valga el pleonasmo) todo lo que constituye el universo real, considerado a su vez en toda su extensión espacio-temporal. Planteadas así las cosas se advierte la existencia de un problema de fondo, que no pretendo en absoluto resolver ( $y$ ni siquiera plantear con todo detalle) en este artículo; es el problema ontológico: ¿Cuáles son los constituyentes de la realidad? ¿Objetos, puntos, sucesos, datos de los sentidos? A la altura actual de la indagación epistemológica parece bastante evidente que, cual. quiera que fuere la actitud que finalmente se adopte respecto del problema ontológico, ha de haber en esa actitud una im. portante componente teórica. Usando el lenguaje neo-kantiano en boga, hemos de admitir que todo enunciado verdadero acerca de la realidad requiere (o implica, en algún sentido) una cierta categorización de la realidad. No es objeto de este artículo proponer ni discutir una tal categorización. Por lo contrario: supongo que se ha elegido una entre tantas posibles (que puede ser, por ejemplo, la que está más o menos implícita en la teoría general de la relatividad); supongo también que tiene sentido hablar de objetos físicos: éstos constituirían el rango de valores de la variable " $x$ " que aparece en la formulación logística de los enunciados legaloides. En consecuencia, esta formulación logística debe ser tomada en sentido semántico, es decir, como abreviatura de locuciones precisas acerca de objetos físicos. Se comprende entonces que la noción de verdad fáctica que se utiliza en este artículo es la más clásica: un enunciado es verdadero si lo que él afirma ocurre en la realidad. También se comprende que esta noción de verdad es independiente de toda noción de verificación: una cosa es que un enunciado sea verdadero, y otra muy distinta es el criterio por el cual llegamos a conocer que es verdadero. Incluso es muy aceptable -en este contexto- la posibilidad de que lleguemos a tener una idea de lo que son los enunciados legaloides (y las leyes naturales) sin que poda. 
mos ofrecer ningún ejemplo seguro de tales enunciados. Esta deficiencia sería lamentable, pero no filosóficamente grave; en efecto, el solo hecho de poder formular con cierta precisión una noción de ley natural que implique la fuerte sospecha de que hay leyes naturales (aunque no podamos conocerlas, o no podamos conocer que lo son) tiene en sí mismo interés filosófico, y ello por dos motivos fundamentales. El primero es obvio, pues tal hecho permitiría hablar significativamente de necesidad física como de algo no idéntico a necesidad lógica, simplemente diciendo que la necesidad física es la que está expresada por las leyes naturales puras (cf. $\$ 3$, 3.5 ) ; el segundo motivo es de carácter histórico-epistemológico: sabiendo (con bastante aproximación) qué son las leyes naturales, y sospechando (en virtud de buenas razones) que ellas existen, adquiere sentido y realce la vieja y honorable tesis histórico-epistemológica del desarrollo científico como aproximación a la verdad; se podría sostener, en efecto, que de las sucesivas teorías científicas pueden derivarse enunciados de carácter fáctico que se aproximan cada vez más (en un sentido que habría que precisar) a leyes naturales, las que aparecerían como representantes conspicuos de La Verdad.

Por los comentarios precedentes queda claro que toda esta discusión sobre leyes naturales se desarrolla sobre el neblinoso (y tormentoso) telón de fondo del problema ontológico y la verdad. Se puede considerar, en consecuencia, que este artículo se inscribe en el capítulo 2 de la filosofía natural.

\section{Objeción sobre los predicados puramente cualitativos}

El requerimiento formal $(e)$ de $\$ 2$, acerca de la mención de objetos particulares, ha sido discutido abundantemente en la literatura científico-filosófica desde hace, por lo menos, un siglo. En [1], Hempel y Oppenheim señalan con particular énfasis las dificultades que surgen cuando se desea precisar con absoluto rigor qué quiere decir que un enunciado no contiene de manera esencial (es decir, no eliminable) referencias a objetos particulares. En estrecha conexión con este 
problema introducen la noción de predicado puramente cualitativo: un predicado es de esta clase "si el establecimiento de su significado no requiere referencia a ningún objeto particular y a ninguna localización espacio-temporal". Hallan luego que esta definición sólo es clara y unívoca si se la formula respecto de un lenguaje formalizado ( $y$ no respecto de un lenguaje natural, como el inglés o el español); y final. mente señalan su objeción fundamental, que estiman insuperable: aun refiriendo la noción de predicado puramente cualitativo a un lenguaje formalizado $\mathrm{L}$, "nuestro problema permanece abierto para los [términos] primitivos del lenguaje, cuyos significados no son determinados por definiciones internas del lenguaje sino más bien por reglas semánticas de interpretación"; y agregan: "Porque deseamos permitir la interpretación de los [términos] primitivos de L por medio de atributos tales como azul, pesado, sólido, más cálido que, pero no por medio de las propiedades de ser descendiente de Napoleón, o un animal ártico, o una estatua griega; y la dificultad es precisamente la de establecer criterios rigurosos para la distinción entre las interpretaciones admisibles y las no admisibles". Observan los autores que de este modo el problema de caracterizar los predicados puramente cualitativos es desplazado al metalenguaje en el que se realiza la interpretación de los términos de L. En efecto: si deseamos dar significado, por medio de reglas semánticas, a los términos primitivos de $\mathrm{L}$, parece adecuado exigir que en la formulación de esas reglas semánticas sólo intervengan, a su vez, predicados puramente cualitativos pertenecientes al metalenguaje. Y si formalizamos adecuadamente el metalenguaje, el problema se desplaza al meta-metalenguaje, y así sucesivamente. $\mathrm{El}$ artículo concluye con un vigoroso acento dubitativo: “... y el problema de una definición adecuada de los predicados puramente cualitativos permanece abierto".

Mi respuesta es la siguiente: en el contexto de este artículo (así como en el del citado trabajo de Hempel y Oppenheim), y en vista del estado actual de las investigaciones filosóficas concernientes a leyes naturales, la exigencia de estos autores 
es excesiva y carece de relevancia; al menos, carece de relevancia en cuanto objeción a una teoría especulativa de leyes naturales. Podría tener relevancia, en cambio, como objeción a una teoría formal que pusiera el acento en los problemas de decidibilidad; pero mi argumento consiste en afirmar que la teoría de leyes naturales se halla aún en una etapa esencialmente especulativa y no está en condiciones de plantearse problemas demasiado formales, y, mucho menos, problemas de decidibilidad. En efecto: teniendo en cuenta la vaguedad esencial que subyace en el problema ontológico señalado en $\S 4$, así como la vaguedad de expresiones muy usuales en la literatura, tales como "cualquier cosa que podría haber satisfecho cierta descripción" (Kneale), "de acuerdo con nuestros supuestos sería posible que una moa viviera más" (Popper), "todos los mundos lógicamente posibles", "todos los mundos que difieren del nuestro, a lo sumo, en las condiciones iniciales" (Popper), resulta sorprendente que se acuse de vaguedad a una expresión tan clara como "no referencia esencial a objetos particulares". Sin ninguna duda, la crítica de Hempel y Oppenheim a esa expresión es lógicamente válida, pero tampoco puede caber ninguna duda de que, si se aplican requisitos metodológicos igualmente estrictos a todas las nociones básicas de la teoría general de leyes naturales, no queda en pie absolutamente nada. Esta teoría está aún en un estado demasiado embrionario como para resistir semejantes pruebas de rigor. Con el rigor lógico sucede algo semejante a lo que acontece en el estudio de los errores de cálculo y de observación: es bien sabido que no se consigue ningún aumento de información fáctica calculando una magnitud con siete decimales si el error experimental en las mediciones de esa magnitud es del orden del segundo decimal; análogamente, ninguna calidad epistemológica se gana al introducir, en lo concerniente a una expresión de una teoría, pautas de rigor notablemente más severas que las que rigen en el cuerpo general de esa teoría. En un contexto en el que se habla libremente de mundos posibles, condicionales subjuntivos (carentes de formalización lógica), realidad (sin pre- 
cisiones ontológicas), y condiciones iniciales (sin definiciones precisas), introducir de pronto una severa exigencia de claridad meridiana para la locución "no requiere referencia a ningún objeto particular y a ninguna localización espaciotemporal", parece excesiva; lo menos que se puede decir es que, en el estado actual de la cuestión, la falta de criterios muy precisos acerca de los predicados puramente cualitativos no quita interés ni vigencia a una teoría sobre leyes naturales. Una cierta prueba pragmática de esta última aserción surge de la confrontación del citado artículo de HempelOppenheim con el ejemplo de las moas y el correlativo concepto de accidente cósmico; en efecto: en aquel artículo se da por sentado (o parece darse por sentado) que el problema de las leyes naturales quedaría resuelto si se clarificara la noción de predicado puramente cualitativo, en tanto que la consideración de los accidentes cósmicos muestra que no es así. Luego, aun dejando sumido en su vaguedad actual el concepto de predicado puramente cualitativo, se lo puede usar para llevar a cabo investigaciones epistemológicas no carentes de interés, como las de Popper y Kneale. Por añadidura, Hempel y Oppenheim utilizan libremente, en el mismo trabajo, dos conceptos que requieren tanto clarificación como el de predicado puramente cualitativo: el de "alcance no limitado" (nonlimited scope) de una sentencia, y la distinción entre leyes fundamentales y derivadas. Esta observación es casi un argumento ad-hominem, pero puede servir como ilustración metodológica.

\section{Objeciones lógico-semánticas}

Las objeciones planteadas en $\operatorname{los} \S \S 4$ y 5 son importantes pero, justamente en una primera aproximación, admiten ser postergadas. Las discusiones finas sobre ontología y verdad, así como sobre definibilidad de predicados, podrán adquirir mayor relevancia cuando se haya construido un primer esquema aceptable para una teoría de leyes naturales. Ahora bien, el esquema propuesto en $\$ 3$ como primera aproximación no es, en definitiva, aceptable: militan en su contra 
algunas objeciones lógicas y lógico-semánticas que pasaré a desarrollar. Estas objeciones, ellas sí, son pertinentes. He expuesto, sin embargo, la primera aproximación de $\S 3$, porque ella permite visualizar con claridad las dificultades contenidas en el plan propuesto, y de este modo ayuda efectivamente a su realización.

La dificultad principal consiste en que, manteniendo el es. quema formal de $\S 3$, pueden aducirse ejemplos que trivializan el concepto de accidente cósmico ( $y$, por ende, el de ley natural) poniendo en evidencia algunas posibilidades total. mente contrarias al espíritu de lo que se desea lograr me. diante el plan propuesto. Los ejemplos trivializadores están conectados con lo que podría llamarse "disyunción promis: cua", que consiste en plantear una alternativa entre dos posibilidades que, desde el punto de vista intuitivo, no tienen nada que ver entre sí; un ejemplo paradigmático de esta técnica de disyunción promiscua está dado por el predicado "verdul" de Nelson Goodman. (El adjetivo "promiscuo", que uso aquí aplicado a la disyunción, fue introducido por Quine para referirse a las teorías de conjuntos. Cf. la locución "conjunto promiscuo".)

Con referencia al ejemplo de las moas de Popper y al esquema formal de $\$ 3$, consideremos el predicado promiscuo "es moa o elefante". Adoptemos también - por vías de ejemplificación- las dos siguientes hipótesis: (i) los virus $\alpha$, que atacaron tan ferozmente a las moas de Popper y las aniquilaron, no se presentaron en el caso de los elefantes; es decir, ningún elefante vivió en presencia de virus $\alpha$; y (ii) todos los elefantes del espacio-tiempo fueron ( $i$ por un aćidente cósmico?) longevos: todos vivieron más de cincuenta años. (No hubo mortandad infantil de elefantes.)

En estas condiciones, abreviemos por " $\mathrm{E} x$ " la sentencia " $x$ es un elefante", y llamemos $\mathrm{L}$ al siguiente enunciado, que es evidentemente legaloide:

$$
(x):[(\mathrm{M} x \vee \mathrm{E} x) \wedge \mathrm{C} x] \supset \sim \mathrm{J} x,
$$

y que se interpreta así: "si $x$ es moa o elefante y vive en am. 
biente carente de virus $\alpha$, entonces $x$ no muere joven (es decir, vive más de cincuenta años)". Obsérvese que la condición de que el antecedente de $L$ no sea universalmente falso (exigida por la definición de enunciado legaloide, cf. 3.1) se cumple gracias a la hipótesis (i) sobre los elefantes. De L se deduce lógicamente:

$$
(x): M x \wedge \mathrm{C} x \supset \sim \mathrm{J} x
$$

que coincide con el enunciado (B) de 3.2. De este modo quedaría probado que el enunciado (A) de 3.2 es un enunciado de accidente cósmico, pero habríamos logrado este resultado por medios intuitivamente repudiables. En efecto: para concluir que la muerte de las moas en plena juventud ha sido accidental, nos basamos esencialmente en el hecho (también accidental, pero sobre todo irrelevante desde el punto de vista intuitivo y científico) de que los elefantes no estuvieran expuestos a la acción de los temibles virus $\alpha$. Lo arbitrario de esta cuestión se hace más patente si se piensa que la misma deducción puede hacerse si hay especies (aunque sean vegetales) cuyos individuos no tropezaron con los virus $\alpha$ y vivieron más de cincuenta años. Pueden intentarse varios remiendos al esquema de $\$ 3$, pero todos los que he examinado resultaron insatisfactorios. La conclusión parece ser la siguiente: si las condiciones impuestas tienen una estructura lógica simple, como las que figuran en 3.2, la devastadora disyunción promiscua permite siempre fabricar ejemplos intuitivamente repudiables. Parece, en consecuencia, que un grado netamente superior de complejidad lógica es inescapable.

La intuición exige que la deducción de (B) se produzca sobre la base de información relevante. Ahora bien, esta exigencia no sólo es difícil de satisfacer, sino que también es difícil de formular con un mínimo de precisión. "Deducir sobre la base de información relevante" parece un enunciado fuertemente conectado con algunos de los más arduos y clásicos problemas filosóficos: la causalidad, los géneros natu. 
rales, la semejanza estructural. Sin embargo, me parece que es posible (y plausible) refinar los métodos usados en $\$ 3$ y resolver el problema sin recurrir a aquellos vastos enigmas filosóficos. Una tentativa provisoria en tal sentido será esbozada en $\$ 7$. Pero el precio que se paga, como ya he insinuado, es el de un abrupto incremento de la complejidad lógica.

\section{Tentativa provisoria de solución}

Lo que ofreceré en esta última parte, de acuerdo con el título que he escogido, es sólo una tentativa, una indicación de posibilidades. Considero muy probable que el esquema que sigue pueda ser "refutado" mediante la exhibición de ejemplos trivializadores, como el que aduje en $\$ 6$ contra la primera aproximación expuesta en $\$ 3$. De todos modos, creo que la metodología que empleo en esta tentativa provisoria puede resultar iluminadora y fructífera en posteriores y más sofisticados acercamientos al tema.

La idea en que se basa esta nueva propuesta ha sido extraída del modus operandi de la ciencia natural (tanto de la física como de la química y de la biología) : se trata de explicar ciertos niveles de organización mediante otros niveles más elementales. En el fondo, es el viejo programa del reduccionismo atomista: explicar los organismos vivientes por la célula, la célula por la molécula, la molécula por el átomo, el átomo por las partículas elementales, etc. (¿etcétera?). Lo que resulta complicado y difícil es dar una versión lógica de este programa reduccionista. Como sigo teniendo in mente el ejemplo de las moas popperianas, y como en él intervienen unos nefastos virus que - presumiblemente - atacaron las células (o ciertas células) de las moas, me servirá de paradigma ( $¡$ no en alguno de los veintisiete sentidos de Kuhn, Dios sea loado!) la situación organismo multicelular-célulavirus. Antes de entrar en los detalles técnicos, desearía exponer brevemente la idea filosófica conductriz, que es ésta: para evitar los efectos trivializadores de la disyunción promiscua y, en este caso particular, para hacer que la estadís. tica sobre los elefantes sea relevante para una estadística 
sobre moas, el procedimiento usual de la ciencia natural consiste en buscar un denominador común: en este caso, la célula. De este modo se termina con la promiscuidad, porque tanto las estadísticas sobre elefantes como las estadísticas sobre moas aparecen como disfraces de una estadística más básica sobre células; se pasa de células a células, y esto tiene un aire más convincente.

Comenzamos por la definición de predicado totalmente explicativo: digo (en términos informales, que luego serán precisados) que el predicado célula es totalmente explicativo del predicado animal multicelular, porque todo lo que se refiere a los animales multicelulares puede explicarse mediante propiedades de las células y relaciones entre células. La formalización de esta idea es la siguiente (pudiendo interpretarse el predicado $\mathrm{C}$ como célula y el predicado $\mathrm{A}$ como animal multicelular):

Definición de predicado totalmente explicativo. Diremos que el predicado $\mathrm{C}$ es totalmente explicativo del predicado $\mathrm{A}$ respecto de un conjunto $E$ (que puede pensarse como el conjunto de los posibles estados de células o conjuntos de células), si se verifican de hecho las tres condiciones siguientes:

(1) $(x): \sim(\mathrm{C} x \wedge \mathrm{A} x)$ (ninguna célula es animal multicelular).

(2) Para todo conjunto $\mathrm{H}$ de individuos que gozan del predicado $\mathrm{C}$ (células) y para todo elemento $e$ de $\mathrm{E}$ (estado), la fórmula $e(\mathrm{H})$ tiene sentido, considerando a $e$ como predicado de $\mathrm{H}$. (Se interpreta como: el conjunto de células $H$ se halla en el estado e).

Antes de formular la tercera y última condición, introduciremos dos notaciones auxiliares:

Relación Pt. Dados dos objetos físicos $x$ e $y$, diremos que se verifica $x$ Pt $y$ si $x$ tiene con $y$ la relación parte-todo, en un sentido físico. Supondremos que el significado de este predicado es suficientemente claro como para resultar de utilidad en el análisis tentativo que sigue. 
$S u f_{C, E}(Q x, P x)$. Diremos que dos condiciones $Q x$ y $P x$ sa. tisfacen $S u f_{C, E}(Q x, P x)$ (puede leerse: $Q$ es una $C \cdot E$ condi. ción suficiente de $P$ ) si se cumplen los siguientes dos réqui. sitos:

(i) Qx tiene la siguiente estructura:

Existen $n$ conjuntos de células $\mathrm{H}_{1}, \ldots \mathrm{H}_{\mathrm{n}}$, tales que sus elementos tienen la relación $P_{t}$ con $x$ y se cum. ple que $e_{1}\left(H_{1}\right) \wedge \ldots . \wedge e_{n}\left(H_{n}\right)$,

donde $e_{i}, \ldots e_{n}$ son elementos de $E$.

(En Qx se pueden detallar también relaciones entre los conjuntos $H_{1}, \ldots, H_{n} v$ el individuo $x$.)

(ii) $\mathrm{Q} x$, en conjunción con enunciados legaloides, implica lógicamente $\mathbf{P} x$.

(En términos informales, estos requisitos establecen que: $\mathrm{Q} x$ afirma que hay conjuntos de células de $x$ en ciertos estados y de tal afirmación puede deducirse $P x$ con ayuda de enunciados legaloides apropiados).

Establecemos ahora la última condición:

(3) Para todo predicado $P$ tal que existen individuos $z$ tales que $\mathrm{A} z$ y $\mathrm{Pz}$ (predicado aplicable en forma no vacía a animales multicelulares) y para todo $x$ tal que $\mathrm{A} x$ y $\mathrm{P} x$, se cumple que $x$ satisface cierta condición $\mathrm{Q} x$, que a su vez verifica $S u f_{\mathrm{C}, \mathrm{E}}(\mathrm{Q} x, \mathrm{P} x)$.

(La aparición de cualquier propiedad $\mathrm{P}$ en un animal multicelular puede explicarse como consecuencia de que ciertos conjuntos de células suyas están en ciertos estados.)

Definición de ampliación de un predicado. El predicado A (animal multicelular) es una ampliación del predicado $\mathbf{M}$ (moa) si de hecho se verifica:

$$
(x): \mathrm{M} x \supset \mathrm{A} x \quad \text { y } \quad \sim(x): \mathrm{A} x \supset \mathrm{M} x
$$

Definición de enunciado de accidente cósmico. Un enuncia. 
do de accidente cósmico es un enunciado legaloide de la forma

$$
(x): \mathrm{M} x \supset \mathrm{J} x \quad \text { (todas las moas mueren jóvenes) }
$$

tal que se cumplen las siguientes condiciones:

(a) Existe una ampliación $\mathrm{A}$ de $\mathrm{M}$ y

(b) Existe un predicado C (célula), totalmente explicativo de $A$ respecto de un conjunto $E$ (estados), y

(c) existe una condición $\mathrm{Q} x$, tal que $S u f_{\mathrm{C}, \mathrm{E}}(\mathrm{Q} x, \mathrm{~J} x)$ y

(d) resultan legaloides los dos enunciados:

(i) $(x): \mathrm{M} x \supset \mathrm{Q} x$

(ii) $\sim(x):(\mathrm{A} x \wedge \sim \mathrm{Q} x) \supset \mathrm{J} x$

(En nuestro ejemplo, Qx podría ser la condición de que una cantidad apreciable de células de $x$ están infectadas por virus $\alpha$. En ese caso, (i) afirmaría que todas las moas tienen células en esa situación y (ii) expresaría que no todo animal multicelular $\mathrm{cu}$ yas células están libres de virus $\alpha$ muere joven.)

Con esta definición de enunciado de accidente cósmico se puede volver al plan de $\$ 3$ y completar la definición de ley natural como allí se hace. Si una definición de este estilo es viable, se habrá dado cuenta de la noción de ley natural sin recurrir previamente a conceptos enigmáticos tales como los de necesidad física, condicional subjuntivo, causalidad, géneros naturales, condiciones iniciales, mundos posibles, etc.

Llamo la atención sobre el papel fundamental que desem. peña en esta exposición el conjunto $E$ (estados). En efecto: ahora no importa que se compare a las moas con otro animal cualquiera, como el elefante; supongamos que todos los animales que no tienen en estado virósico sus células relevantes para morir jóvenes (es decir, los A que no son Q), sean elefantes. La condición (ii) dice entonces que algunos de tales elefantes no mueren jóvenes. No se trata ahora de una esta- 
dística promiscua, en la que se infieren propiedades de las moas a partir de propiedades de los elefantes: ahora hay un concepto unificador que hace que en cierto sentido impor. tante los elefantes sean relevantes para las moas; ese concepto unificador es el de célula. Pero el concepto de célula es el que tenemos intuitivàmente in mente: en la definición propiamente dicha aparece un predicado abstracto $\mathrm{C}$; para que este predicado $C$ sea realmente unificador (como es su ejemplo paradigmático "célula") hay que evitar que el mismo $\mathrm{C}$ sea promiscuo. Esta no promiscuidad de $\mathrm{C}$ es lo que está asegurado por la presencia del conjunto $\mathrm{E}$ de los estados, los cuales son aplicables tanto a células de moa como a células de elefante. En otras palabras: lo que hace que las células de las moas y las de los elefantes estén relacionadas en forma relevante, y no promiscua, es el hecho de que tenga sentido asignar los mismos estados a esas diferentes células. En otros términos, y desde un punto de vista ligeramente distinto, podría decirse que el hecho de que haya un único conjunto de estados aplicables por igual a células de moas y a células de elefantes, hace que todas estas células formen parte de “un mismo género natural” (el género célula, por supuesto).

De este modo se ve que el argumento de promiscuidad trivializadora esgrimido en $\$ 6$ contra la primera aproximación no vale ahora frente a la nueva propuesta; al menos, no vale en la forma simple con que lo he presentado. Pero no tengo ninguna seguridad de que esta tentativa provisoria de solución sea inmune a formas más refinadas de promiscuidades trivializadoras, o a otros contraejemplos malignos.

\section{REFERENCIAS}

[1] Hempel, Carl and Oppenheim, P. "Problems of the Concept of General Law", en Danto and Morgenbesser (eds.), Philosophy of Science. World Publishing Company, 1966.

[2] Popper, Karl R. La lógica de la investigación cientifica. Madrid: Editorial Tecnos, 1962.

[3] Simpson, Thomas M. “Necesidad natural y mundos posibles: Observaciones sobre Popper", Ciencic e Investigación, tomo 27, No. 7, julio 1971. 
The purpose of this article is to give an account of the concept of natural law. It is known that the expression "natural law" is used in scientific-philosophical literature in various senses, all of them more or less interrelated. In this paper the concept of natural law is characterized as a pattern of nature's behaviour, in opposition to natural law as a valid statement in the context of a theory. Therefore, the main question raised is: what feature would a law have that would describe the universe's real behaviour? As we can see, this is a question that has an ontological component. But we can also see that this is not the most ontological version of the problem of natural laws. Ignoring the ontological problem in a strong sense, an ontological problem in a weak sense is left: still admitting that a law is a simple statement, in other words, a certain linguistic entity, what logical-semantical-grammatical feature must a statement have to deserve the name of a description of the universe's behaviour? The last question leads us to establish some necessary conditions for calling a statement a natural law. The following, expressed in an informal language, are such conditions:

(a) Veracity: natural laws must be true statement about nature's behaviour;

(b) Universality: natural laws must be valid statements in general, that is, not restricted to a determinate space-time zone of the universe;

(c) Non-contingency: natural laws must be statements free of every shade of contingency. Of course, unless the meaning of "contingency" is explained, this condition is useless.

The precise purpose of this paper is to establish the logical form of natural law according to the intuitive conditions previously adopted.

(d) Communicability: natural laws must be theoretically communicable statements in the following sense: every intelligent being acquainted with the meaning of our general terms and who dominates the grammar of our language must be in condition to understand the statements we called natural laws.

In virtue of $(c)$ and $(d)$, we can draw a well-known conclusion, namely that natural laws must not contain terms which designate particular objects in essential (not eliminable) form. Actually, the 
essential occurrence of the naime of a particular object seems to make the universe's behaviour depend on the existence of an object. This can be thought of as an excessive dose of contingency, if we think - by way of an example - that every isolated object is in itself contingent.

On the other hand, in virtue of the condition (b), specifications of time and space cannot figure in essential form in the statement of a natural law.

From conditions $(a)-(d)$ the following basic conditions can be derived:

(e) No essential mention should be made of particular objects;

( $f$ ) No essential use of numerical specifications of space-time character should be made.

The primitive conditions $(a),(b),(c),(d)$, and the derived ones, $(e)$ and $(f)$, are interesting, but not enough. Two fundamental problems emerge: the problem of the purely qualitative predicates of Hempel-Oppenheim, and the problem of the cosmic accidents of Popper-Kneale-Simpson.

By cosmic accident, we understand something that describes a contingent natural regularity. This is very clearly exposed by Popper's classical example about the moas in New Zealand. In spite of being a strictly universal statement, "every moa dies before reaching fifty years of age", its contingent character, put into evidence by a natural law, would not be produced in determinate conditions, objectively different from those that were really given.

We must keep in mind that a cosmic accident is a fact expressable by means of a strictly universal statement, and that it provokes some problems which are tentatively resolved in this paper. The problem raised here is that in order to characterize a cosmic accident we need the previous introduction of the concept of natural law. This seems circular, but as we shall see it is only apparent. Therefore, the approach to the possible solution is based on the concept of "legaloid statement".

Of what has been said above a reasonable plan to solve the abovementioned problem arises. It consists of the following: (1) to give a characterization, with the help of the conditions $(a)-(f)$, of a previous concept for a law, which is the legaloid statement; (2) to give a characterization, among the legaloid statements, of those which express a cosmic accident, exemplified paradigmatically by the example of the Popperian moas; (3) to define as a natural law every legaloid statement which is not a cosmic accident (and that has no other minor inconvenience). 
What is first offered is a first approximation to that scheme, which seemingly translates the intuitive ideas exposed above into formal terms, and which outlines the profound difficulties, of logical-semantic character, that arise in the development of this plan.

A formal and precise definition of legaloid statement and of cosmic accident statement are given and also commented on in detail. Later some commentaries on ontology and truth are made, because the definition of a legaloid statement requires that such a statement be true inasmuch as it refers to reality.

The formal requirement (e) has been discussed abundantly in scientific-philosophical literature for the last century. In connection with the difficulties that arise when we want to define precisely what is meant when we say that a statement does not contain essential references to particular objects, we can use the notion of purely qualitative predicate: a predicate is purely qualitative "if the establishment of its meaning does not require reference to a particular object and to a space-time localization".

Hempel and Oppenheim think the following difficulties to be unavoidable: even if the notion of purely qualitative predicate is referred to a formal language $L$, "our problem remains open for the primitive [terms] of the language, whose meanings are not determined by internal definitions of language, but by semantic rules of interpretations". These authors observe that this way of dealing with the problem of characterizing the purely qualitative predicates is to push it into the metalanguage in which the interpretations of terms of $L$ are realized. But if we adequately formalize the metalanguage, the problem is pushed into the meta-metalanguage, and so on.

The answer for this is the following: in the context of this paper (and also in the cited work of Hempel and Oppenheim) and in view of the present state of the philosophical investigations concerning natural laws, the demand of these authors is excessive and lacks relevance, that is, lacks relevance as an objection to a speculative theory of natural laws. It could have some relevance as an objection to a formal theory which puts the accent on problems of decidability; but the argument in this paper consists of establishing that the theory of natural laws is still in an essentially speculative stage, and that it is not in condition to trace problems that are too formal. Therefore, we have to leave the concept of purely qualitative predicate in its present state and vagueness although it still can be of interest to epistemological investigations.

The objections outlined above are important but must be postponed in the first approximation. The fine discussions on ontology and truth, as well as on the definability of predicates, would be more relevant in a scheme acceptable for a theory of natural laws. 
There are some logical and logico-semantical objections to the scheme proposed in $\$ 3$, some of which are relevant to the purposes of this paper. These are connected with what is called, in Quine's terms, "promiscuous disjunction", which consists of proposing an alternative between two possibilities that, from an intuitive point of view, have nothing to do with each other.

After examining Popper's example once again, the author of this paper gives a solution to the problem which is not only provisional but also tentative, an indication of possibilities.

It is possible that the scheme proposed could be refuted by means of trivial examples such as those given against the first approach in § 3. It is the author's belief, however, that the methodology used in this provisional, tentative solution could be illuminating and fruitful in other more sophisticated approaches to the subject.

The idea on which the new proposal is based has been obtained from the "modus operandi" of natural science (especially physics, and also chemistry and biology): it is meant to explain some organizational levels by means of other more elementary levels. In the course of this new approach, new concepts are defined, such as a totally explainable predicate, which permits the definition of other concepts, and finally the concept of cosmic accident statement, upon which the definition of natural law is based. The plan developed in this paper enables us to avoid enigmatic concepts such as physical necessity, subjunctive conditional, causality, etc.

According to the author, the argument of trivial promiscuity against the first approximation has no validity against the second one. There is no guarantee, however, that the tentative, provisional solution is immune to other more refined kinds of promiscuities, or other perverse counterexamples.

(Summary by Arturo Cisneros) 